Classification

Physics Abstracts

$68.10-44.25$

\title{
Thermal coupling in layered convection: evidence for an interface viscosity control from mechanical experiments and marginal stability analysis
}

\author{
Ph. Cardin, H.-C. Nataf and Ph. Dewost \\ Département de Géologıe, Ecole Normale Supérıeure, 24 rue Lhomond, 75231 PARIS cedex 05, \\ France
}

(Recelved 18 September 1990, revsed 15 February 1991, accepted 11 March 1991)

\begin{abstract}
Résumé. - Nous étudıons la convectıon dans un système à deux couches liquides superposées: huıle au silıcone au-dessus de glycérol, en partıculıer. Nous avons mené deux types d'études (1) En laboratoıre, nous analysons le rôle dynamıque de l'ınterface, en induısant, par un système de deux cylındres en rotation inverse, des mouvements en rouleau dans une des couches. Grâce à des pallettes d'aluminıum en suspension dans les liquides, nous suivons les lignes de courant et mesurons les vitesses de part et d'autre de l'interface. Nous obtenons des résultats très différents selon le sens de rotation des cylındres (convergence ou divergence), et selon l'amplitude de la vitesse imposée. Nous en déduisons l'existence d'une résıstance interfaciale quı est du même ordre que la contrainte visqueuse volumique, et que l'on peut associer à une forte viscosité d'interface. (2) Nous avons étudié numériquement la stabilité marginale d'un système convectif a deux couches. Nous utilısons les propriétés physiques du système huile silicone/glycérol, et nous analysons en détail l'influence de la viscosité d'interface. Nous passons en revue le rôle de la variation des épansseurs des deux couches, de la deformabilité de l'interface, de la dépendance en température de la tension interfaciale et du rapport de densité. Nous montrons qu'aucun de ces effets, s'll reste dans une plage réalıste, n'est capable de modifier le couplage préféré au seuil: ıl s'agıt du couplage "mécanıque", dans lequel les courants descendants de la couche du haut sont situés au-dessus des courants montants de la couche du bas. Par contre, l'introduction d'une viscosité d'interface, compatıble avec les observations expérimentales, modıfie le type de couplage: le couplage "thermique" (courant montant au-dessus de courant montant) devient le mode préféré Ce résultat lève la contradıctıon qui exıstait jusqu'alors entre l'observation du couplage "thermıque" dans les expériences de convection en laboratorre, et la prédıctıon de couplage "mécanıque" des études de stabılıté margınale et des sımulatıons numériques.
\end{abstract}

\begin{abstract}
Convection in a two-layer system (sllicone oll over glycerol, in particular) is studied. Two types of studies have been performed (1) In the laboratory, we analyse the dynamical role of the interface, by inducing roll-like motions in one of the liquid layers, using a system of two inversely rotatıng cylinders. Streamlines and velocities on both sides of the interface are measured, by observing suspended aluminum particles. The results strongly depend upon the sense of rotation of the cylinders (convergence or divergence), and upon the amplitude of the imposed velocity. This implies an interface strength, which is of the same order as the volumic viscous strength, and that can be associated to a large interface viscosity. (2) A numerical study of convective marginal stability in a two-layer system has been performed. The physical properties of the silicone oll/glycerol system are used, and special attention is given to the influence of interface viscosity. The role of varying the depth ratio, the deformation of the interface, the temperature-dependence of interface tension, and the density ratio is reviewed It is shown that none of these effects, when realistic values are taken, is able to modify
\end{abstract}


the preferred type of couphng at the threshold: it remains "mechanical" coupling (downwellings in the upper layer remain above uprisings in the lower layer). However, the type of coupling is changed when the interface viscosity is introduced, with values compatible with the experimental observations: "thermal" coupling (uprisings above uprisings) becomes the preferred convective mode. This result removes the contradiction there was until now between the observation of "thermal" coupling in laboratory convection experiments, and the prediction of "mechanical" coupling obtained from marginal stability analysis and numerical experiments.

\section{Introduction.}

Rayleigh-Benard convection in a system of two superposed liquids has received considerable attention. Geophysicists have become interested in this problem because convection in the Earth's mantle might be layered, with the upper mantle convecting above the lower mantle [1]. Physicists have also been attracted by layered convection, because of the expected wealth of possible dynamical behaviours. In particular, layered convection has been the framework for a major theoretical study of coupling with non-linear interaction terms [2].

Even at the threshold of convection, the system exhibits a wide variety of behaviours, as demonstrated by a series of marginal stability analyses [3-9]. Richter \& Johnson [5] discovered a regime with oscillatory critical convection, when the densities of the two fluids are almost equal. Honda [4] noted the existence of two superposed marginal stability curves: one corresponds to "mechanical" coupling (i.e. convection rolls rotate in a gear-like fashion, with upnsings of the upper layer above downwellings of the lower layer), the other one corresponding to "thermal" coupling (with uprisings above uprisings). There are therefore several possible marginally stable states, analogous to the different overtones for convection in a single layer. The essential difference from the single layer case is that these two curves are very close to each other, and they correspond to two different types of coupling. Honda [4] found that "mechanical" coupling was the mode that should develop first (i.e., it has the lowest Rayleigh number), when the two liquids have similar properties. The other curve, corresponding to "thermal coupling", is also marginally stable, but requires higher values of the Rayleigh number. Other contributions have dealt with the role of interface deformation, and interfacial tension. A rather thorough analysis has been conducted by Rasenat et al. [9]. These authors-show that, for suitable choices of the properties of the two liquids, convection can be oscillatory at the threshold, the oscillations being between a "mechanical" and a "thermal" extreme. Finally, Wahal \& Bose [8] introduced several additional interface parameters, including interface viscosity, but did not report on the type of coupling produced.

Finite amplitude numerical experiments have also been carried out in two dimensions $[10,11]$. They show that "mechanical" coupling remains stable for individual Rayleigh numbers up to $10^{6}$. Thermal coupling is only obtained in cases where the viscosities of the two liquids are very different (by more than a factor of 30). Three-dimensional numerical experiments [12] have confirmed and extended these results.

Laboratory experiments have also been performed. In two previous studies $[13,14]$ we reported observations for two pairs of working liquids: silicone oil over glycerol, and two different silicone oils. In both cases, the experumental results are in contradiction with the numerical predictions: "mechanical" coupling is not stable in the lab (even when induced at the threshold), and "thermal" coupling is observed instead. Nataf et al [13] show that the effects of the interface deformation and interface tension cannot explain this paradox. They postulate that some kind of interface viscosity is present, and propose that it would favor "thermal" coupling.

In the present contribution, we report on two separate developments that give support to this 
interpretation. First, we designed some mechanical entrainment experiments, in order to evaluate the mechanical role of the interface for our working liquids. We observed that the interface played a very significant role in the transmission of the velocities from one liquid to the other, as much as to totally inhibit the transmission of motion in some cases. Second, convinced of the existence of some interface viscosity, we conducted a marginal stability analysis, using the physical properties of our working fluids and including this new ingredient. In the analysis, we examine the role of several effects, such as interface deformation, interface tension, and interface viscosity, in order to better assess their actual influence on the type of coupling. The most striking result is that "thermal" coupling takes over "mechanical" coupling when interface viscosity is introduced.

This resolves our paradox, and demonstrates that Rayleigh-Benard convection can be used as a good revealer for the presence of interface viscosity.

\section{MECHANICAL ENTRAINMENT EXPERIMENTS.}

Since we suspected that interface viscosity was responsible for the observed thermal coupling in our Rayleigh-Benard convection experiments, we tried to assess more carefully the mechanical role of the interface. The measurement of interface viscosity and elasticity is not yet something standard, and we found no values in the literature for the particular pairs of liquids we used. Although several methods have been proposed for these measurements [15-17], there is no simple and well established method.

From a mathematical point of view, interface viscosity enters the balance of the tangential stresses at the interface, which is written as [18]:

$$
\mu_{t} \frac{\partial u_{t}}{\partial z}-\mu_{b} \frac{\partial u_{b}}{\partial z}=\Sigma+(\eta+\epsilon) \frac{\partial^{2} u}{\partial x^{2}}
$$

where $\mu_{t}$ and $\mu_{b}$ are the volumic shear viscosities of the top and bottom liquids, and $u_{t}$ and $u_{b}$ their horizontal velocities (here in the $x$ direction), $\Sigma$ the interface tension, $\eta$ and $\epsilon$ the dilatational and shear interface viscosity, respectively. All right hand side terms specifically refer to interface (molecular) properties, and are ignored in most studies of layered convection. Interface viscosity is introduced when a thin film of surfactants is present at the interface. Note that both the interface tension and the interface viscosity depend upon the local concentration of surfactants. Interface viscosity could also exist at the interface between pure liquids [19].

One of the difficulties in measuring interface viscosity is that for it to play a role, the motion at the interface must have a divergent (or convergent) component $\left(\frac{\partial^{2} u}{\partial x^{2}} \neq 0\right.$ ). We designed our own apparatus for assessing the mechanical role of the interface. It is not really quantitative, but its geometry is very close to that of the thermal convection experiments.

\section{The experimental set-up.}

The principle of the apparatus is the following: we force roll-like motions in one of the liquid layers, and we observe the resulting induced motions in the other layer. The actual set-up is shown in Figure 1. The two liquids are contained in a lucite tank, whose inner dimensions are the same as for the convection experiments: 50 (height) $\times 125 \times 250 \mathrm{~mm}^{3}$. The interface lies at midheight. Roll-like motions are generated by two horizontal brass cylinders placed at mid-depth of one of the liquid layers. The axes are horizontal and parallel to the short side of the tank. The spacing between the axes is $25 \mathrm{~mm}$, equal to the depth of each liquid layer. The two cylinders, 


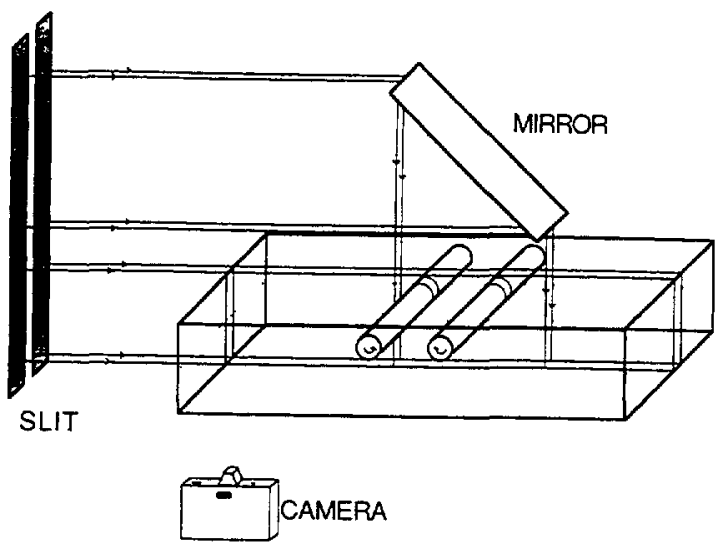

F:g. 1. - Perspective view of the experimental set-up. A thin vertical slice of light is emitted by the stroboscopic lamp on the left (slit). It enters the rectangular lucite tank, and lightens the liquid. Part of the light beam is reflected down by a mirror at $45^{\circ}$. Long exposure pictures are taken of the illuminated aluminum flakes in the superposed liquids. The interface between the two liquids is indicated by a dashed line. The brass cylinders rotate around their horizontal axis with opposite vorticity. The arrows indicate the sense of motion : here a "convergent" case. The different components are not drawn to scale.

whose diameter is $13.9 \mathrm{~mm}$, rotate in opposite senses, driven by a single stepping motor, with appropriate shafts.

Aluminum flakes are introduced in each liquid. A stroboscope lightens a slice of the liquids, perpendicular to the cylunders. A mirror is used to reduce the extent of the cylinders shadows. Streak photographs are taken on a camera aligned with the cylinders. Streamlines and velocities are measured from these photographs.

Two pairs of liquids have been used: 47V500 Rhone Poulenc silicone oil above glycerol (as In the convection experiments of Nataf et al [13] ), and olive oil above diluted glycerol. Bulk properties, for the first pair are given in Table I, together with the propertues of the two silicone oils (47V100 and 550) that were used to make the silicone "light" over silicone "dense" convection experiments of Cardin \& Nataf [14]. These will be used in the marginal stability analysis, in the second part of this paper. Within each pair, the volumic viscositıes of the two liquids are almost equal.

\section{Silicone oil over glycerol.}

2.1 OBSERVATIONS. - Figure 2 shows the streamlines observed for a $3.3 \mathrm{~mm} / \mathrm{s}$ tangential velocity on the cylinders (placed in the upper layer). This velocity is comparable to typical convective velocities. In Figure $2 \mathrm{a}$, the forced motion is convergent at the interface, between the cylinders, as indicated by the white arrows (we call this a convergent case), while in Figure $2 b$ the forced motion is divergent. The first order result is that motion is transmitted across the interface. A closer look at the velocities shows that the velocity beneath the interface in the induced rolls is comparable to that of the lateral induced rolls in the upper layer. At first sight, the interface therefore plays no mechanical role per se.

However, we observe that the cell pattern depends upon the entrainment velocity. Figure 3 shows two pictures. The top one is a convergent case, with tangential velocity on the cylinder of $33 \mathrm{~mm} / \mathrm{s}$. By comparing with Figure $2 \mathrm{a}$, we observe that two side counter-rotating cells are now 
Table I. - Physical propertzes of the liquids.

\begin{tabular}{|c|c|c|c|c|c|}
\hline & S.I. Units & Glycerol & $\begin{array}{l}\text { Rhodorsil } \\
\text { silicone } \\
47 V 500 \\
\end{array}$ & \begin{tabular}{|c} 
Rhodorsil \\
silicone \\
550 \\
\end{tabular} & $\begin{array}{l}\text { Rhodorsil } \\
\text { silicone } \\
47 V 100 \\
\end{array}$ \\
\hline$k$ Thermal conductivity & $\mathrm{W} \mathrm{m}^{-1} \mathrm{~K}^{-1}$ & 0.294 & 0.16 & 0.146 & 016 \\
\hline$\rho$ Density $\left(25^{\circ} \mathrm{C}\right)$ & $\mathrm{kg} \mathrm{m}^{-3}$ & $1.26 \times 10^{3}$ & $0.97 \times 10^{3}$ & $1.07 \times 10^{3}$ & $0.97 \times 10^{3}$ \\
\hline$C_{p}$ specific heat & $\mathrm{J} \mathrm{kg}^{-1} \mathrm{~K}^{-1}$ & $2.62 \times 10^{3}$ & $146 \times 10^{3}$ & $1.50 \times 10^{3}$ & $146 \times 10^{3}$ \\
\hline$\kappa$ Thermal diffusivity & $\mathrm{m}^{2} \mathrm{~s}^{-1}$ & $0.89 \times 10^{-7}$ & $1.13 \times 10^{-7}$ & $0.91 \times 10^{-7}$ & $1.3 \times 10^{-7}$ \\
\hline $\begin{array}{c}\nu \text { Kinematic viscosity } \\
\left(25^{\circ} \mathrm{C}\right)\end{array}$ & $\mathrm{m}^{2} \mathrm{~s}^{-1}$ & $7.45 \times 10^{-4}$ & $4.99 \times 10^{-4}$ & $1.25 \times 10^{-4}$ & $1.00 \times 10^{-4}$ \\
\hline$\alpha$ Thermal expansion & $K^{-1}$ & $4.9 \times 10^{-4}$ & $945 \times 10^{-4}$ & $7.5 \times 10^{-4}$ & $945 \times 10^{-4}$ \\
\hline $\begin{array}{c}\Sigma \text { Interfacial tension } \\
\left(25^{\circ} \mathrm{C}\right)\end{array}$ & $\mathrm{N} \mathrm{m}^{-1}$ & \multicolumn{2}{|c|}{$25 \times 10^{-3}$} & \multicolumn{2}{|c|}{$<5 \times 10^{-3}$} \\
\hline $\begin{array}{c}\frac{\partial \Sigma}{\partial T} \text { Temp. derivative } \\
\text { of } \Sigma\end{array}$ & $\mathrm{N} \mathrm{m}^{-1} \mathrm{~K}^{-1}$ & \multicolumn{2}{|c|}{$13 \times 10^{-4}$} & & ? \\
\hline
\end{tabular}

present in the lower layer.

Even more striking is the role of the sign of the velocity. Figure $3 \mathrm{~b}$ is a divergent case, with the same absolute entrainment velocity as in Figure 3a. The counter-rotating cells are not present in the divergent case.

A careful analysis of the streamlines reveals another difference $:$ in the convergent case (Figure $2 \mathrm{a})$, there is a triangular zone beneath the interface between the cylinders, where velocities are more reduced than in the divergent case (Figure $2 \mathrm{~b}$ ). This shows up in horizontal profiles of the vertical velocity derived from the streak photographs. Figure 4 compares two such profiles obtained in the convergent and divergent cases. Both run horizontally at the level of the centers of rotation of the induced rolls of the lower layer, about $12 \mathrm{~mm}$ below the interface. The absolute velocity is clearly smaller in the convergent case.

2.2 INTERPRETATION. - The observation that is most suggestive of a specific mechanical role of the interface is the lack of symmetry between the convergent and the divergent cases. No volumic property can explain this observation. Indeed, the only 'sense' is given by the gravity vector, which plays no role in these purely mechanical experiments, in the volume of the liquids. The deformation of the interface does depend upon the sense of rotation: the interface is uplifted 

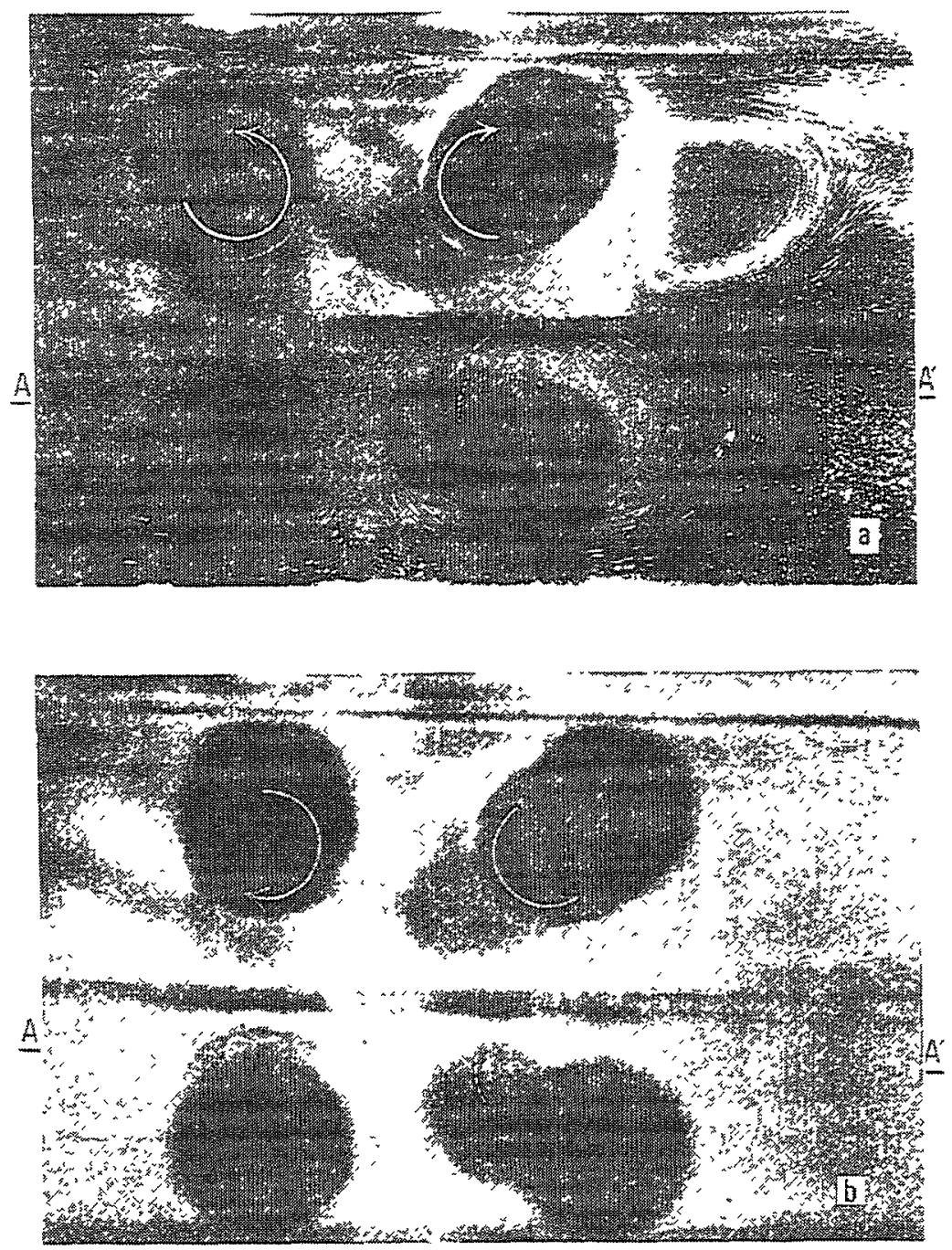

Fig. 2 - Streak photographs for two entranment experıments with tangential velocity $V=3.3 \mathrm{~mm} / \mathrm{s}$. The. upper liquid is silicone oil, and the lower one is glycerol. The two rotating cylinders are at mid-depth in the upper layer. (a) Convergent case: the motion between the cylinders is up. The aluminum flakes show roll-like motion in the lower layer. (b) Divergent case: the motion between the cylinders is down. Note that the vertical velocity in the entrained rolls below the interface is smaller in the convergent case than in the divergent case. The end of the cylinders are seen as light circles, with the sense of motion superimposed. The dark circles are corks on the lucite frame; there is no cylinder in the bottom liquid layer. The stroboscopic light had 6 pulses, each 4 seconds long. The total exposure time is $\mathbf{4 0}$ seconds. Lines AA' are the positions of the two horizontal profiles shown in Figure 4.

between the cylinders in the convergent case. However, the deformation is very small. We can estimate its amplitude $h$ from a simplified balance of the normal stress at the interface:

$$
\left(\rho_{t}-\rho_{b}\right) g h=\mu_{t} \frac{\partial w_{t}}{\partial z}-\mu_{b} \frac{\partial w_{b}}{\partial z}
$$

where $g$ is the acceleration due to gravity, $\rho$ are the densities, and $w$ the vertical velocities. We 

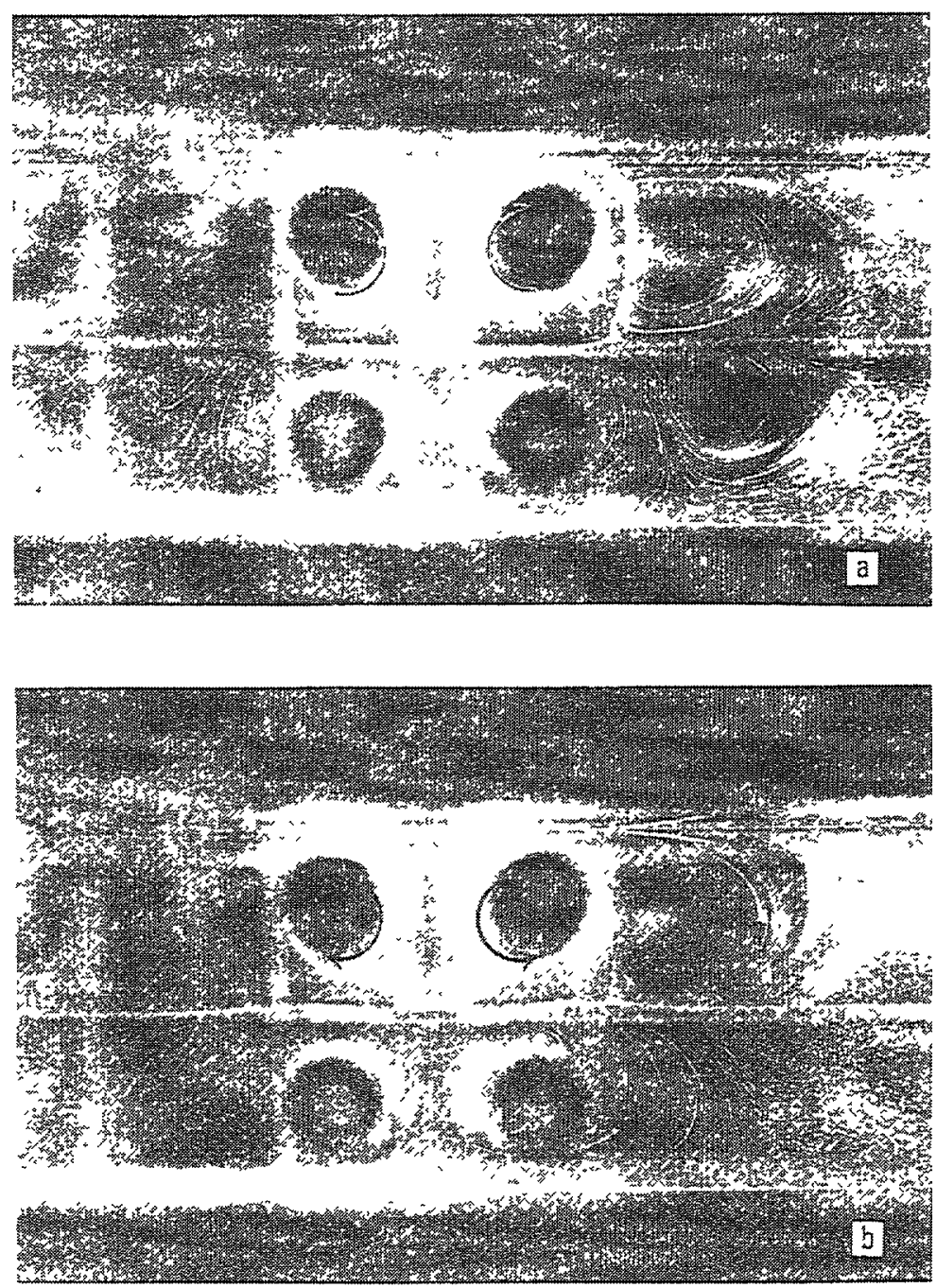

Fig. 3. - Same as Figure 2 but with an entrainment velocity of $33 \mathrm{~mm} / \mathrm{s}$ (a) Convergent (b) Divergent Contınuous lightning. The exposure tume is 60 seconds. Note that two counter-rotating rolls on the sides in the bottom layer are visible in the convergent case, but not in the divergent one. On the contrary, the streamlines are "rounded" near the central stagnation point, in the convergent case.

have neglected the interface tension terms that would reduce $h$, and will look for an upper bound by setting the induced dynamical pressure $\mu_{b} \frac{\partial w_{b}}{\partial z}$ to zero. We then get:

$$
h<\frac{\mu_{t}}{\left(\rho_{t}-\rho_{b}\right) g} \frac{V}{d / 2}
$$

where $V$ is now the imposed tangential velocity on the cylinder, and $d$ is the thickness of a liquid layer. This yields an upper bound deformation of $0.1 \mathrm{~mm}$ when $V=3.3 \mathrm{~mm} / \mathrm{s}$, as for the runs of Figure 2. This value is in agreement with the small deffection observed in the streak photographs. 


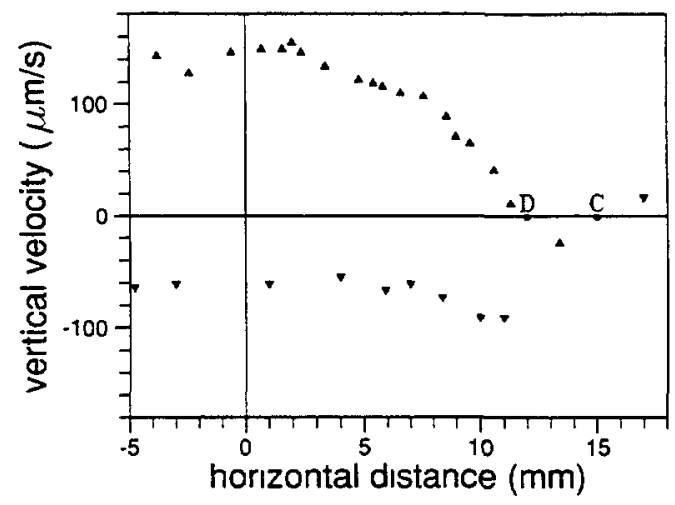

Fig. 4. - Horizontal profiles of the vertical velocity in the lower liquid layer. The profiles (whose positions are indicated by lines AA' in Figure 2) run through the motionless centers of rotation of the entrained rolls. Their horizontal position is noted $\mathrm{C}$ and $\mathrm{D}$ for the convergent and divergent cases, respectively. The horizontal distance $x$ is measured from the vertical line that lies at equal distance from the two rotatıng cylinders. The up-triangles are the up-going velocities of the divergent case, the down-triangles are for the convergent case. Note that the vertical velocity at $x=0$ is much smaller in the convergent case than in the divergent case.

Its effect on the velocity field is very small. Furthermore it should probably have an effect opposite to the one we observe, by inducing slightly larger velocities in the lower layer in the convergent case.

Inertial effects would not explain the lack of symmetry either : the Reynolds number is written as :

$$
\mathcal{R} e=\frac{V d}{\nu}
$$

where $\nu=\mu / \rho$ is the kinematic viscosity. We get a value of 0.9 for the high velocity runs of Figure 3 , so that inertial effects are negligible.

We conclude that the lack of symmetry can only be explained through mechanical properties of the interface. Note, however, that a constant interface viscosity does not explain it either. We propose a simple model that accounts, qualitatively, for all the observations mentioned above. Suppose that some unknown surfactant is present at the interface. Its concentration depends upon velocities at the interface : the molecules are swept away from places where flow is divergent at the interface, and accumulate in zones of convergent flow. We further assume that high concentrations of surfactants lead to a large interface viscosity. Then the interface tends to act as a barrier to stress transmission where flow is convergent on the interface.

This explains the triangular zone of reduced coupling between the cylinders in the convergent case. On the contrary, the interface is cleaned between the cylinders in the divergent case, and the surfactants pile up on the sides, thereby inhibiting the formation of the induced side counterrotating rolls. Finally, increasing the entrainment velocity results in narrowing the zones of high concentration, which permits an overall better transmission of motion.

We have chosen here to present an explanation based on concentration-dependent interface viscosity. One could as well propose one with a concentration-dependent interface tension, with high tension in zones of high concentration. Indeed, this is the classical explanation for contamination of liquid bubbles rising into water $[20,21]$. We have no experimental evidence that would dismiss one or the other.

To get a more quantitative assessment of the mechanical role of the interface would require 
knowledge of the velocity field induced when the interface is purely passive. We do not know it in this geometry. However, if we assume that the interface viscosity is zero in the zones of low concentration, we can derive a lower bound for the interface viscosity in the zones of high concentration, by comparing the vertical velocity profiles of the convergent and divergent cases. Since the vertical velocity drops by a factor of about 2.5 in the convergent case, we deduce a rough lower bound for the interface viscosity in high concentration zones:

$$
\eta+\epsilon \sim 06 \mu d \sim 0.6 \times 10^{-2} \mathrm{~kg} \mathrm{~s}^{-1}
$$

At this stage, we think that we have demonstrated the existence of some interface viscosity of the order of $\mu d$ in our mechanical experiments. Since the liquids tested are the same as in the Rayleigh-Benard convection experiments that featured thermal coupling, and that the velocities, the geometry, and the experimental procedure are similar in the two cases, we think that this interface viscosity is present as well in the convection experiments. We will show in the next section that it is indeed responsible for thermal coupling to be preferred over mechanical coupling.

It remains that we do not know what is at the origin of the interface viscosity. We have postulated the existence of surfactants, but we ignore their exact nature. The lucite tank was cleaned with ethyl alcohol, and the liquids were poured directly from the manufacturer's container, but no special care was taken for avoiding contamination. We do not think that the aluminum particles introduced are the contaminating agent. They did tend to cluster at the interface, but not in a contunuous fashion. In addition, these aluminum particles have been shown not to be tensioactive in other experiments [22].

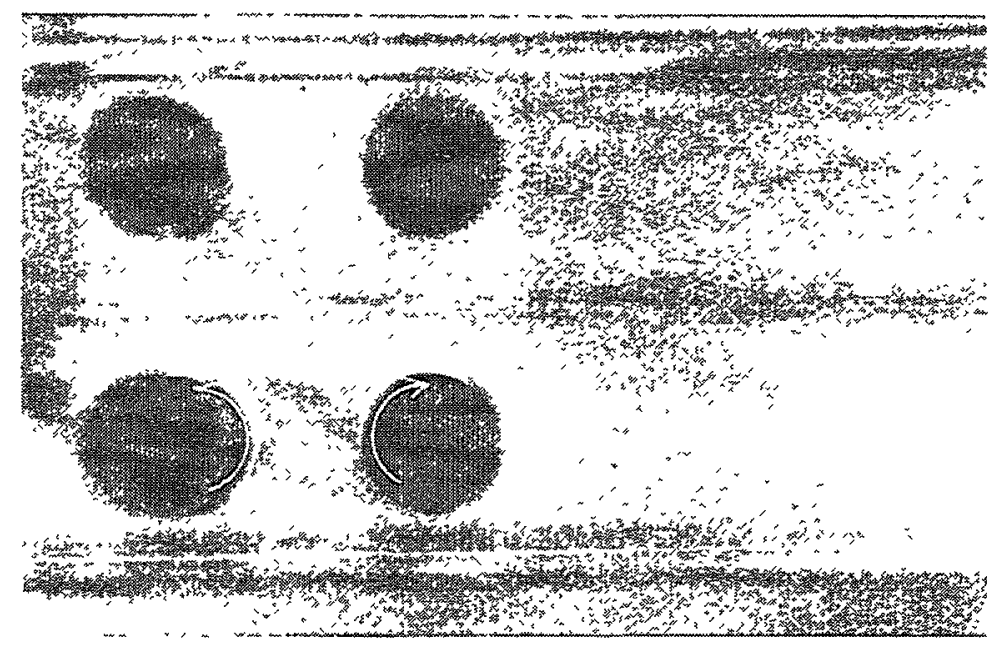

Fig. 5. - Streak photograph for olive oll over diluted glycerol. The rotating cylinders are now in the lower layer. The tangential velocity is $3.3 \mathrm{~mm} / \mathrm{s}$ The exposure time is 60 seconds. No motion is observed in the upper (olive oll) layer. 


\section{Olive oil over diluted glycerol.}

47V500 silicone oil is made of rather long polymeric chains. This is known to enhance interface effects. It was therefore suggested that using a vegetable oil, with much shorter chains, could remove the interface viscosity effect. We experimented with olive oil over a mixture of $85 \%$ glycerol and $15 \%$ water (so that the volumic viscosities of the two liquids are identical; we measured $\nu=$ $68 \times 10^{-5} \mathrm{~m}^{2} / \mathrm{s}$ at $25^{\circ} \mathrm{C}$ for olive oil). The rotating cylinders were placed in the lower layer. As shown in Figure 5, the result was rather disappointing: whatever the entrainment velocity up to $65 \mathrm{~mm} / \mathrm{s}$, no motion was transmitted across the interface! This means that the entrainment shear stress is entirely balanced by interface tension or viscosity. This yields an overall interface strength up to $\tau=\mu_{t} \frac{\partial u_{t}}{\partial z} \simeq 07 \mathrm{~kg} \mathrm{~m}^{-1} \mathrm{~s}^{-2}$, significantly larger than for the silicone oil over glycerol case, a somewhat surprising finding.

\section{MARGINAL STABILITY ANALYSIS.}

The above experimental study demonstrates the need for introducing interface viscosity, in order to model properly the mechanical interaction of the two superposed liquid layers. We will now introduce it in a study of convective marginal stability. Marginal stability in a layered system has been studied by many investigators, and papers by Rasenat et al. [9] and Wahal \& Bose [8] are rather exhaustive. We will restrict our analysis to the two pairs of liquids for which we have experimental data, and in particular to the silicone oil over glycerol system. We will show that classical ingredients do not predict thermal coupling, but that when interface viscosity is introduced, thermal coupling does dominate over mechanical coupling.

We start with a brief recall of the equations and method, with special emphasis on the modelisation of the interface.

\section{Equations and method.}

1.1 Equations. - We consider two superposed immiscible liquids. The average position of the interface is at $z=0$. The thicknesses of the two liquid layers are $d_{t}$ and $d_{b}$, the indices $t$ and $b$ standing for top and bottom, respectively. Each layer is governed by the classical equations [23] that derive from the conservation of mass, momentum, and energy, and an equation of state of the form:

$$
\rho_{\mathbf{\imath}}=\rho_{0,2}\left(1+\alpha_{\imath}\left(T-T_{0}\right)\right)
$$

where $\rho$ is the density, $\alpha$ is the coefficient of thermal expansion, $T$ is the temperature, and $\iota$ equals $t$ or $b$. We use the Boussinesq approximation in the Navier-Stokes equation. The equations are non-dimensionalized, using the properties of the bottom layer with scales $d_{b}$ for length, $d_{b}^{2} / \kappa_{b}$ for time, and $\beta_{b} d_{b}$ for temperature, where $\kappa$ is the thermal diffusivity, and $\beta$ the temperature gradient. The equations in the bottom layer then are written as:

$$
\begin{aligned}
\operatorname{div} \mathbf{u}_{b} & =0 \\
\operatorname{Pr}^{-1}\left(\frac{\partial}{\partial t}+\mathbf{u}_{b} \nabla\right) \mathbf{u}_{b} & =\nabla p_{b}+\mathcal{R} a T_{b} \mathbf{k}+\Delta \mathbf{u}_{b} \\
\frac{\partial T_{b}}{\partial t} & =\mathbf{u}_{b} \mathbf{k}+\Delta T_{b}
\end{aligned}
$$


where $\mathbf{u}$ is the non-dimensional velocity vector, and $k$ the unit vector in the vertical direction. The Rayleigh and Prandtl numbers are defined using the material properties of the bottom layer:

$$
\mathcal{R} a=\frac{\alpha_{b} g \beta_{b} d_{b}^{4}}{\kappa_{b} \nu_{b}}, \quad \operatorname{Pr}=\frac{\nu_{b}}{\kappa_{b}}
$$

For the top layer, the equations become:

$$
\begin{aligned}
\operatorname{div} \mathbf{u}_{t} & =0 \\
\operatorname{Pr}^{-1}\left(\frac{\partial}{\partial t}+\mathbf{u}_{t} \cdot \nabla\right) \mathbf{u}_{t} & =\rho \nabla p_{t}+\alpha \mathcal{R} a T_{t} \mathbf{k}+\nu \Delta \mathbf{u}_{t} \\
\frac{\partial T_{t}}{\partial t} & =\beta \mathbf{u}_{t} \cdot \mathbf{k}+\kappa \Delta T_{t}
\end{aligned}
$$

where $\rho, \alpha, \nu, \beta, \kappa$ are the "top over bottom" ratios:

$$
\rho=\frac{\rho_{t}}{\rho_{b}}, \quad \alpha=\frac{\alpha_{t}}{\alpha_{b}},
$$

To the motionless solution of these equations are added sinusoldal pertubations in vertical velocity $w$ and temperature:

$$
\begin{aligned}
& w_{t}=W_{\imath}(z) \exp (\imath a x+\sigma t) \\
& T_{i}=\Theta_{\imath}(z) \exp (\imath a x+\sigma t)
\end{aligned}
$$

where $a$ is the horizontal wave number of the pertubation, and $\sigma$ the complex growth factor. The perturbations are supposed small, and second order terms are ignored. One then gets the usual system of linear equations:

$$
\begin{aligned}
\left(D^{2}-a^{2}-\frac{\sigma}{P r}\right)\left(D^{2}-a^{2}\right) W_{b} & =\mathcal{R} a a^{2} \Theta_{b} \\
\left(D^{2}-a^{2}-\sigma\right) \Theta_{b} & =-W_{b} \\
\left(D^{2}-a^{2}-\frac{\sigma}{\nu P r}\right)\left(D^{2}-a^{2}\right) W_{t} & =\frac{\alpha}{\nu} \mathcal{R} a a^{2} \Theta_{t} \\
\left(D^{2}-a^{2}-\frac{\sigma}{\kappa}\right) \Theta_{t} & =-\frac{\beta}{\kappa} W_{t}
\end{aligned}
$$

where $D=\frac{d}{d z}$

We will look for values of $\mathcal{R} a, \sigma$ and $a$, such that this system of equations has non-zero solutions. Only solutions with $\sigma=0$ or $\sigma=\imath \omega$ with $\omega$ real will be kept (i.e. marginally stable solutions). Note that although there are two layers, only one Rayleigh number must be introduced. Here, we have chosen to define the Rayleigh number using the properties of the bottom layer. Once a solution found for this Rayleigh number, one can of course compute the corresponding Rayleigh number for the upper layer, knowing the "top over botom" parameters.

We now have to solve this double set of sixth order differential equations. For this, we need the boundary conditions.

1.2 BOUNDARY CONDITIONS. - We have 12 boundary conditions to specify. The upper and lower boundaries provide 3 each, and 6 are provided by the interface between the two lquids. 
1.2.1 The top and bottom boundanes. - Both boundaries are assumed rigid and isothermal, as in the laboratory setting. We get:

$$
\begin{array}{ll}
\text { at } z=-d_{b} & \text { at } z=d_{t} \\
W_{b}=0 & W_{t}=0 \\
D W_{b}=0 & D W_{t}=0 \\
\Theta_{b}=0 & \Theta_{t}=0
\end{array}
$$

1.2.2 The interface. - This is where most of the physics comes in. Indeed, we will see that depending upon the physical hypotheses made, the convective solutions change drastically. We consider here the general case of a deforming interface, with interface tension and viscosity. Let $\xi$ be the interface deformation around the mean position $z=0$ The kinematic condition at the interface then implies that it should be of the form:

$$
\xi=\Xi \exp (i a x+\sigma t)
$$

with

$$
\sigma \Xi=W_{b}(z=0)=W_{t}(z=0)
$$

Also at $z=0$, we have:

- Continuity of tangential velocity:

$$
D W_{b}=D W_{t}
$$

- Continuity of tangential stress:

$$
\left(a^{2}+D^{2}\right) W_{b}=\mu\left(a^{2}+D^{2}\right) W_{t}-M a(\Theta-\Xi) a^{2}-N_{v_{2}} D W a^{2}
$$

- Continuity of normal stress:

$$
\frac{1}{a^{2}}\left(D^{2}-3 a^{2}-\frac{\sigma}{P r}\right) D W_{b}=\frac{\mu}{a^{2}}\left(D^{2}-3 a^{2}-\frac{\sigma}{\nu P r}\right) D W_{t}+\left(\mathcal{R} a_{\rho}+a^{2} S c\right) \Xi
$$

- Continuity of temperature:

$$
\Theta_{b}=\Theta_{t}
$$

- Continuity of heat flux:

$$
D \Theta_{b}=k D \Theta_{t}
$$

where $k$ is the top over bottom ratio of thermal conductivities.

Other dimensionless numbers are:

The Marangoni number:

$$
M a=-\frac{\partial \Sigma}{\partial T} \frac{\beta_{b} d_{b}^{2}}{\nu_{b} \kappa_{b} \rho_{b}}
$$

The Schmidt number:

$$
S c=\Sigma \frac{d_{b}}{\nu_{b} \kappa_{b} \rho_{b}}
$$

A “density Rayleigh number":

$$
\mathcal{R} a_{\rho}=\frac{\left(\rho_{b}-\rho_{t}\right)}{\rho_{b} \alpha_{b} \beta_{b} d_{b}} \mathcal{R} \boldsymbol{a}
$$


And the interface viscosity number:

$$
N_{v i}=\frac{\nu_{2 n t}}{\nu_{b} d_{b}}
$$

where $\nu_{i n t}$ is the "kinematic" interface viscosity:

$$
\nu_{\imath n t}=\frac{(\eta+\epsilon)_{\imath n t}}{\rho_{b}}
$$

In the following, we will sometimes use the approximation that the deformation of the interface is negligible.

We then have:

$$
\Xi=W_{b}(z=0)=W_{t}(z=0)=0
$$

Note that the normal stress is then no longer continuous at the interface, and the corresponding continuity equation is dropped.

1.3 THE AlgorithM. - We use a classical shooting method and fourth order Runge-Kutta integration, such as described, for example, in Krueger et al. $[24,25]$. In each layer, the sixth order linear differential equation has a 6-dimensional solution space. The general solution $F_{z}(z)$ (with $\imath=t$ or $b)$ is a vector with components $\left(W, D W, D^{2} W, D^{3} W, \Theta, D \Theta\right)$. It is found as a linear combination of six independent basis functions $f_{3}$ :

$$
F_{z}=\left[a_{1} f_{1}+a_{2} f_{2}+.+a_{6} f_{6}\right]_{z}
$$

The $f_{j}$ functions are computed by integrating (with 30 to 100 steps) the vectors $(1,0,0,0,0,0)$, $(0,1,0,0,0,0), \ldots,(0,0,0,0,0,1)$, from $z=d_{\imath}$ to $z=0$. In order to determine the particular solution $F_{1}$ in each layer, we have to determine the six coefficients $a$, . The boundary conditions (II.8) imply that $a_{1}=a_{2}=a_{5}=0$ There remain three unknown $a$, in both layers. The six boundary conditions at the interface (II.10 to II.15) provide the required constraints. Non-trivial solutions are obtained when the determinant of the resultung system is zero. For each fixed value of $a$, we look for values of $\mathcal{R} a$ and $\sigma$ that cancel the determinant. When $\sigma=0$, this is done by converging to a zero determinant using a Newton-Raphson method. When $\sigma$ is pure imaginary, we look for the minimum of the modulus of the complex determinant. The eigenvalues and eigenfunctions are then computed.

A numerical problem arises when a complex (i.e., oscillatory) solution bifurcates into two real (i.e., steady) solutions, as the wave number is increased or decreased. The determinant is then the small difference of large values, and the solution for $\mathcal{R} a$ and $\sigma$ is poorly constrained. For a given precision threshold, it is even possible to obtain a complex 'solution' where there should not be any, as shown by the existence of two real solutions for the same wave number. Since we are not especially interested in these regions of the solution space, we have kept both solutions, with no special attempt to resolve the ambiguity.

1.4 CHECK OF THE ALGORITHM. - We checked the algorithm by reproducing the marginal stability curves obtained by Honda [4] for the case

$$
k=d=\kappa=\beta=\alpha=\mu=\rho=1, M a=N_{v \imath}=S c=0 \text {. }
$$

We mentioned that two curves are obtained in an $\mathcal{R} a$ versus wave number plot. The lower one corresponds to "mechanical" coupling, and the upper one to "thermal" coupling. Note that this case corresponds to two completely identical liquid layers. This may be somewhat confusing, since the system can then be viewed as one single layer. Indeed, the lowest curve, which 
exhibits mechanical coupling is simply the first overtone of a single layer, as determined by Pellew \& Southwell [26]. However, the upper curve, corresponding to thermal coupling (as well as the suppression of the fundamental of a single layer) can only be obtained if the deformation at the interface is assumed to be negligible. This violates the condition $\rho=1$. In fact, this condition should be seen as meaning: "the density ratio is large enough for the layering to be stable and the deformation of the interface to be negligible $\left(\mathcal{R} a_{\rho} \rightarrow \infty\right)$, but is close to 1 in other respects". The previous case displays no overstability (i.e. oscillatory solutions). We therefore also checked our algorithm against another case, treated by Rasenat et al [9], which exhibits overstability.

For that case also, which has:

$\alpha=\kappa=\rho=1\left(\right.$ but $\left.\mathcal{R} a_{\rho} \rightarrow \infty\right), \mu=0.1, \beta=25, d=025, M a=N_{v \varepsilon}=S c=0$,

we reproduce adequately the published curves.

\section{Marginal stability results.}

We now look at the effect of different terms in the interface continuity equations. We start from the simplest case with no deformation, no interface tension, and no interface viscosity. Then, we include one of these effects, which we want to understand, and analyse the change in the marginal stabulity curves and eigenfunctions.

The other parameters are taken equal to their experimental values, as listed in Table II. Most results are for the silicone oil over glycerol case; the silicone oil "light" over silicone oil "dense" case is only introduced when investigating the role of interface viscosity. :

In order to clarıfy the presentation, we always show marginal stability curves in the same format: Rayleigh number $\mathcal{R} a$ versus non-dimensional wave number a. Also drawn is the non-dimensional pulsation $\omega$ of the oscillations, where the marginal solution is oscillatory. The "reference" curve with no interface effect is recalled in all figures (solid line). We sometimes "label" the curves with a drawing of the vertical velocity eigenfunction, which best describes the prevailing coupling.

2.1 VARYING THE DEPTH RATIO. - Rasenat et aL [9] showed that, for certain combinations of parameters, convection can be overstable. They note, as did Mutabazzi et al. [25] for a related problem, that this happens when the "individual" Rayleigh numbers of each layer are similar. We are interested in finding this regime for our silicone oil over glycerol system (see also Cardin \& Nataf [14]). Since physical properties are fixed, this can only be achieved by changing the depth ratio $d$. Indeed we find a region of overstability for $d=0.76$ (the glycerol (bottom) layer is thicker than the silicone (top) one). The corresponding marginal curves are shown in Figure 6 . The region of overstability is for values of a between about 2 and 2.9. The case $d=0.76$ (with no interface properties added) will be our reference case in the following. Also drawn are cases $d=0743$, when the upper and lower curves barely separate, and $d=0.72$. Note that the set of upper curves for large a all join to a single curve, and so do the set of lower curves for small a. They all correspond to convection that is driven by the lower layer. By this we mean that convection is more vigorous in the lower layer, and that the upper layer is almost passively dragged, as we will show below. On the contrary, the two other sets correspond to convection driven by the upper layer, as can be seen from the insets showing the vertical velocity eigenfunctions. Had we chosen a non-dimensionalisation based on the parameters of the upper layer, we would have obtained the complementary pattern shown in Figure 7. In this figure, the curves that correspond to convection driven by the lower layer are now well separated, and the corresponding eigenfunctions do display a more vigorous lower layer. The physics of the two figures is the same: only the way to present the results has changed. Although the curves are very close, the corresponding vertical velocity eigenfunction can be fairly different. Note, for example, that the upper curve for $d=072$ is 
Table II. - Numencal data.

\begin{tabular}{|c|c|c|}
\hline Non-dimensional $\frac{\text { top }}{\text { bottom }}$ & $\frac{47 V 500}{\text { glycerol }}$ & $\begin{array}{l}\text { silicone "light" } \\
\text { silicone "dense" }\end{array}$ \\
\hline $\begin{array}{l}k \text { Thermal conductivity } \\
\qquad(\beta=1 / k)\end{array}$ & 0.54 & 1.10 \\
\hline$\rho$ Density $\left(25^{\circ} \mathrm{C}\right)$ & 077 & 091 \\
\hline$\kappa$ Thermal diffusivity & 1.27 & 143 \\
\hline $\begin{array}{c}\nu \text { Kinematic viscosity } \\
\left(25^{\circ} \mathrm{C}\right)\end{array}$ & 067 & 08 \\
\hline$\alpha$ Thermal expansion & 192 & 126 \\
\hline$M a$ Marangoni number & -40 & $?$ \\
\hline$S c$ Schmidt number & $5 \times 10^{4}$ & $10^{4}$ \\
\hline Pr Prandtl number & 8400 & 1800 \\
\hline$R a_{p}$ Density Rayleigh number & $1.4 \times 10^{9}$ & $7 \times 10^{8}$ \\
\hline$N_{v z}$ Interface viscosity number & $>1$ & $?$ \\
\hline
\end{tabular}

associated with thermal coupling.

The overstability regime occurs when the two sets of marginal stability curves would cross. As mentioned by Rasenat et al [9], the oscillations are between a rather thermal coupling extreme and a mechanical coupling extreme. Indeed, if we examine the vertical velocity at mid-depth of each layer, we find that they display a sinusoidal amplitude variation with time, but with a phase difference. When the two velocities have the same sign, the coupling is thermal, and it is mechanical when the velocities are of opposite sign. This example illustrates the diversity of convection in a two-layer system, with competition not only between mechanical and thermal couplings, but also between the strengths of convection in the two layers.

We note that, for our particular liquids, convection always should start with mechanical coupling. Oscillations are not expected to be observed, since the overstability region does not yield the lowest Rayleigh number. 


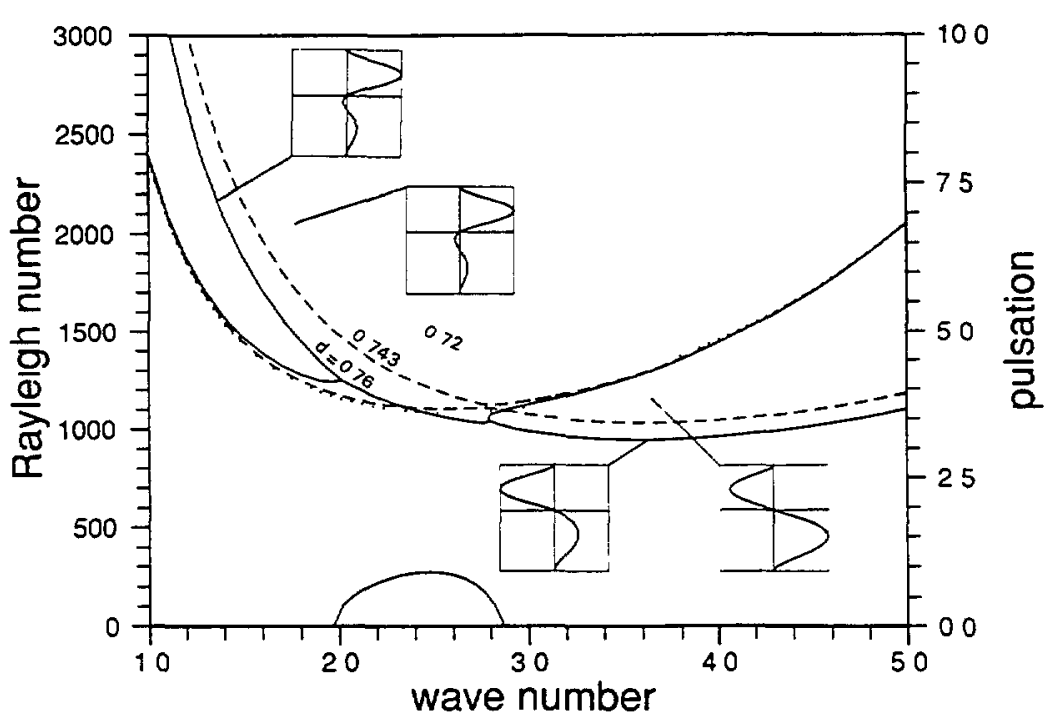

Fig. 6. - Marginal stability curves computed in the silıcone oll/glycerol system, for different depth ratios $d=d_{t} / d_{b}$. Other values as in Table II. No interface effect $\left(M a=N_{v z}=\mathcal{R} a_{p}^{-1}=S c=0\right)$. The Rayleigh number $\mathcal{R} a$ is plotted against the non-dimensional honzontal wave number a. The solid line is for our reference case with $d=0.76$. It displays two superposed branches at low and large values of the wave number. For a between 2 and 2.9 , we get a single marginal stability curve, which is overstable. The corresponding non-dimensional pulsation is drawn at the bottom, with its axis on the right side. It seems that the pulsation does not vanish at $a=28$, where two stationary branches are actually found This is a numerical artefact (see text). The insets are the vertical velocity eigenfunctions. They show that the lower curve for large a is mechanical coupling driven by the top layer, while the upper curve for small a is a quasithermal coupling also driven by the top layer. The dashed curves are for $d=0743$ Note that the two curves almost meet in the middle. The dotted curves are for $d=0.72$

2.2 DEFORMATION OF THE INTERFACE. - In the previous section, the interface was assumed to be underformed. We now relax this assumption and investigate the effect of the deformation of the interface. Deformation is multiplied by a sum of two terms in the continuity equation of normal stress (II.13): one scales with $\mathcal{R} a_{\rho}$, the other with $S c$. From Table II, we see that the former is of the order of $10^{8}$ and the latter of about $10^{4}$. We will therefore neglect the second term, and look at the effect of $\mathcal{R} a_{\rho}$. Figure 8 gives the results for $\mathcal{R} a_{\rho}$ values of $10^{3}, 10^{4}, 10^{5}$. For the largest value, the marginal stability curves are undistinguishable from the no-deformation case. We can therefore already confirm that the deformation of the interface is negligible in our experiments, as mentioned by Nataf et al. [13]. It is interesting however to analyse the results with low $\mathcal{R} a_{\rho}$ values. For $\mathcal{R} a_{\rho}=10^{4}$, the large wave number end of marginal stability is unaffected, but overstability now extends to very low a values, where the pulsation shows a peak. For $\mathcal{R} a_{\rho}=10^{3}$, convection is predicted to be oscillatory at the threshold. The pattern of oscillations is shown in Figure 9. The vertical velocity does not vanish at the interface, which implies that the interface actually deforms with time, as predicted by Richter \& Johnson [3]. We note however that the results of Figure 8 and 9 indicate a smooth transition from the interface dominated oscillations to the oscillations between thermal and mechanical extremes, which we discussed previously. For even lower values of $\mathcal{R} a_{\rho}$, the critical Rayleigh number tends to 171, and the critical wave number to 1.7 , which correspond to convection of the whole system as a single layer (we recall that the non-dimensionalisation is based on the lower layer). Note that the density ratio only enters in equation (II.13), multiplied by the interface deformation. Therefore, it has no effect in regimes other than overstable. 


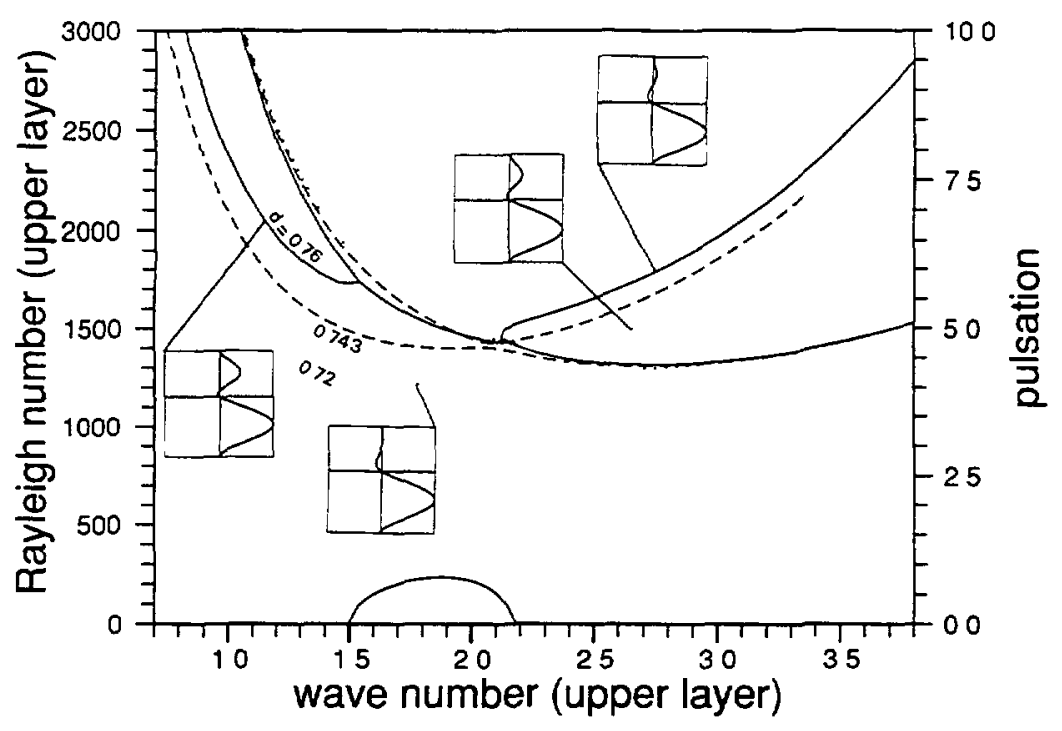

Fig. 7. - Same results as in Figure 6, but with the Rayleigh number, wave number, and pulsation nondimensionalized using the properties of the upper layer. The curves that correspond to convection driven by the lower layer now separate, and show a wde variety of etgenfunctions.

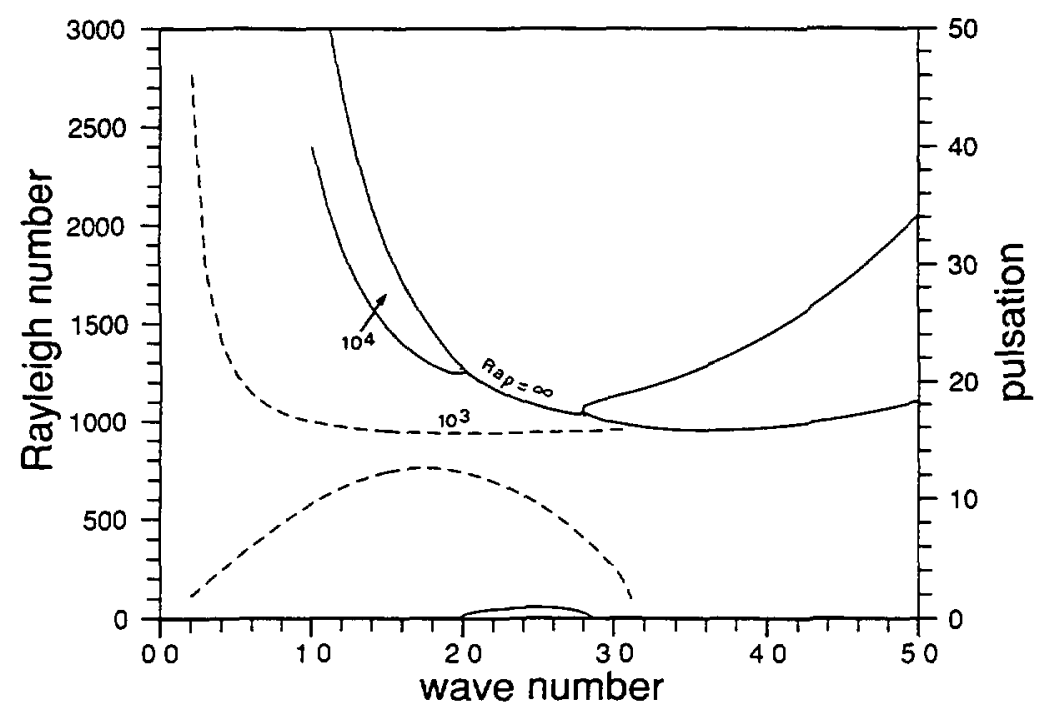

Fig. 8. - Marginal stability with interface deformation. The solid line is our reference case with no interface deformation. The dotted line is the overstable curve (and the corresponding pulsation curve at the bottom) for $\mathcal{R} a_{\rho}=10^{4}$. The dashed curve is for $\mathcal{R} a_{\rho}=10^{3}$ : overstability extends over a large range of wave numbers (the axis starts at $a=0$ ). Note that the range on the pulsation axis has been increased by a factor of 5 as compared to Figure 6. 


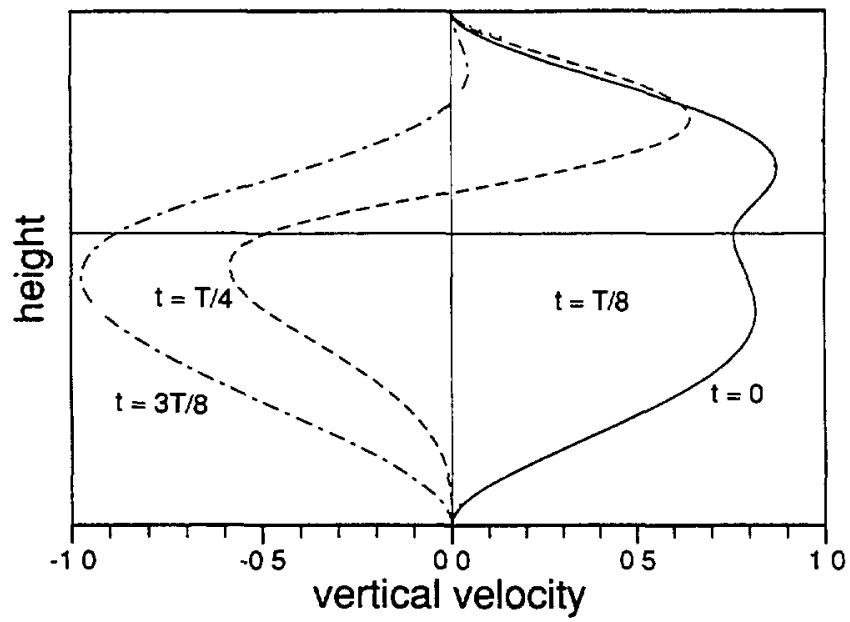

Fig. 9. - Vertical profiles of the vertical velocity at different times for the overstable regime with $\mathcal{R} a_{\rho}=10^{3}$ $(\mathcal{R} a=938, a=2.5, \omega=9.5)$. The horizontal line is the position of the interface at rest. Note that the velocity at the interface can get quite large, and that the coupling goes from rather thermal (same sign in both layers) to rather mechanical (velocities are of opposite sign), depending on the time within the oscillation cycle. (the eigenfunction for $t=T / 2$ is the same as for $t=0$, with the opposite sign).

2.3 MARANGONI CONVECTION. - We now let interface tension be a function of temperature. This introduces a coupling between the velocity field and the temperature field, the so-called Marangoni effect. For most liquids, the Marangoni number is positive. Wahal \& Bose [8] report that the Marangoni effect is rather stabilizing: it tends to decrease the extent of the overstability region. Figure 10 shows that this is also true in our case for moderate Marangoni numbers. Indeed, for a Marangoni number as low as 10, the overstability region has completly disappeared. However, Nataf et al. [13] measured an increase of interface tension with temperature in the silicone oil over glycerol system, which yields a negative Marangoni number. The case with a value of -200 , which is typical, is also shown in Figure 10. The dashed curves in the insets are the profiles of the horizontal velocity eigenfunctions. Because of incompressibility, the horizontal velocity is equal to the derivative of the vertical velocity with respect to depth. This time, the overstability region is quite wider. The upper branch for large wave number now corresponds to mechanical coupling, but with a much reduced horizontal velocity at the interface. The slope discontinuity of the horizontal velocity profile is reminiscent of experimental results of Villers \& Platten [27].

Note that depending upon the phase shift between the temperature and the velocity fields, the Marangoni effect is resistive or driving. This is well seen in the horizontal velocity profiles, and it explains why the marginal stability branches tend to get closer or to move away from each other, depending upon the sign of the Marangoni number.

2.4 INTERFACE VISCOSITY, AND THERMAL COUPLING. - None of the effects we have studied so far - depth ratio, interface deformation, temperature-dependent interface tension - enables thermal coupling to be the preferred mode of convection. In fact, for the most realistic parameter values, stable mechanical coupling is expected. Our only hope for explaining thermal coupling as observed by Nataf et al [13] now rests upon the effect of interface viscosity.

We now introduce this effect. We will examine both the silicone oil over glycerol case and the silicone oil "light" over silicone oil "dense" case. 


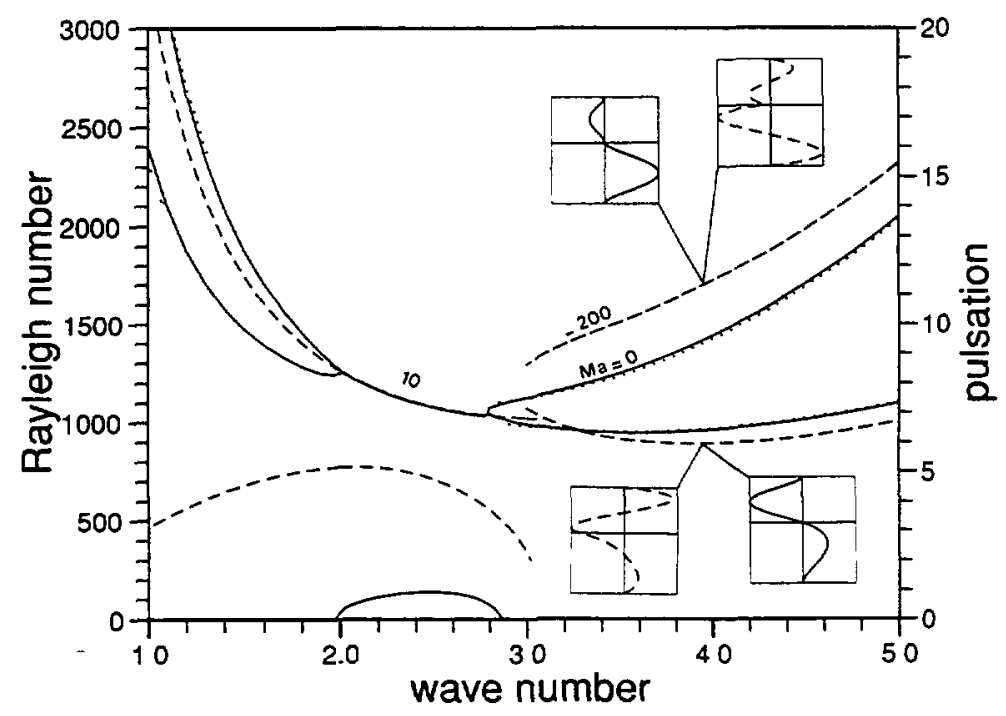

Fig. 10. - Marginal stability with temperature-dependent interface tension. The solid lines are our reference case with zero Marangonı number. The dotted curves are for $M a=10$. Note the disappearance of the overstability region. The dashed curve is for $M a=-200$. The overstability region occupies the low wave number end. Two branches are found for larger wave numbers. Both display mechanical coupling, as shown by the insets. The lowest Rayleigh number is obtained for $M a=-200$. The dashed line in inset is the horizontal velocity eigenfunction. It shows a clear slope discontinuity.

2.4.1 Silicone oil over glycerol - Figure 11 summarises the effect of interface viscosity on our reference case. For values of $N_{v i}$ less than 1, we observe a small widening of the overstability region, and an increase of the Rayleigh numbers of all branches. For values of $N_{v z}$ larger than 1, things get quite different: the overstability region disapears; the lower curve does not move much, but the upper curve moves to higher Rayleigh number values quite significantly. Wahal \& Bose [8] looked at the effect of interface viscosity. They mention that for $N_{v z} \geq 10^{-1}$, it tends to reduce the overstability region. We find here a more contrasted behaviour. However, the most interesting finding rests with the eigenfunctions. While the lowest curve was always associated with mechanical coupling so far, the lowest curve for $N_{v \imath} \geq 2$ corresponds to thermal coupling, as shown in Figure 11. It is important to note that, although the lower curve has not moved much, the eigenfunction that goes with it has changed dramatically. Figure 12 displays in more detail the mechanical coupling vertical velocity eigenfunctions for increasing values of $N_{v_{1}}$. Note that for $N_{v z}>1$, this eigenfunction is not associated with the lowest curve. Also drawn is the thermal coupling eigenfunction for $N_{v i}=10$. As $N_{v i}$ increases, the stress transmission at the interface becoming less efficient, the lower layer is not "dragged" anymore; the vertical velocity in the lower layer increases, as the increase in critical Rayleigh number allows it. Horizontal velocity is shown in Figure 13. It tends to zero at the interface as $N_{v s}$ increases. The liquid layers see the interface more and more as the rigid boundaries at the top and bottom. For even larger $N_{v i}$ values, the marginal stability curves do not move anymore, and the eigenfunctions are very similar, until the interface is actually seen exactly as a rigid boundary.

2.4.2 Silicone ou "light" over silicone oil "dense". - These two oils have been used by Cardin \& Nataf [14]. The properties of the two silicone oils (47V100 and 550) that were used to make them are given in Table I and the corresponding ratios in Table II. In the experiments, thermal coupling 


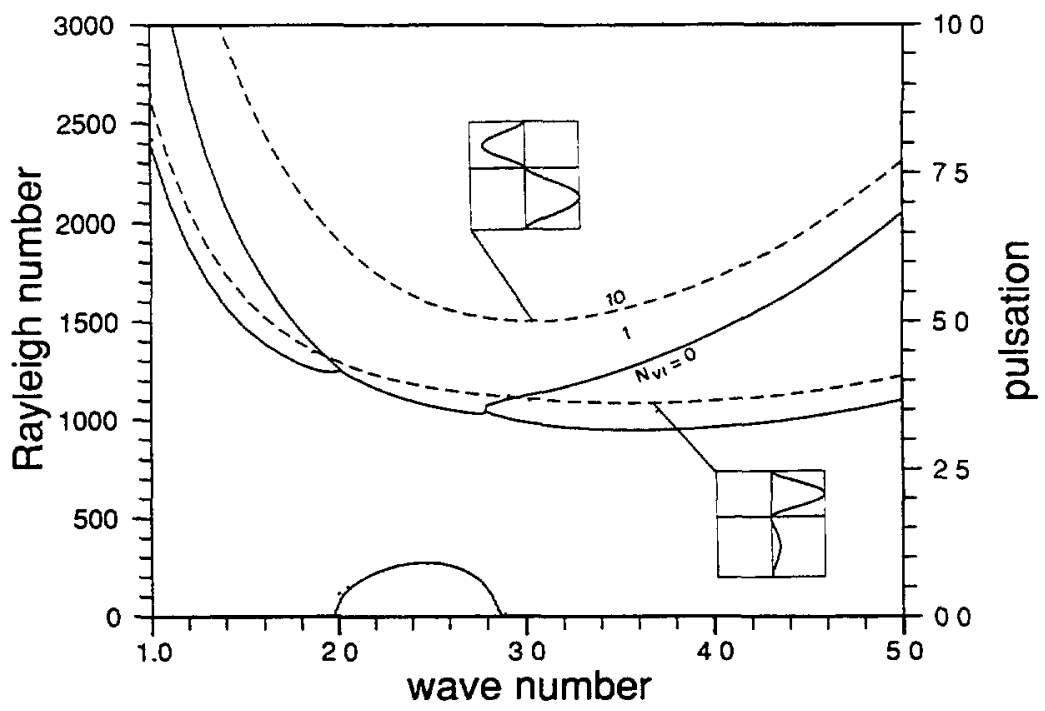

Fig. 11. - Marginal stability with interface viscosity. The solid lines are for our reference case with zero $N_{v z}$ number. The dotted curves are for $N_{v i}=1$, and the dashed curves for $N_{v i}=10$. The vertical velocity eigenfunctions show that the lowest cntical Rayleigh number is for thermal coupling in that case.

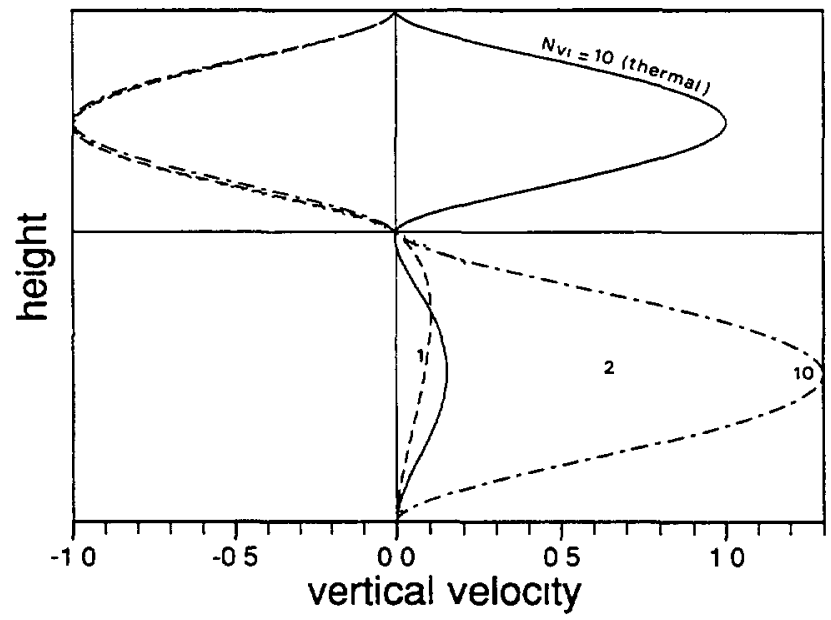

Fig. 12. - Vertical profiles of the vertical velocity for different $N_{v i}$ values. Solid hne: Thermal coupling (preferred) for $N_{v z}=10$; chain line: mechanical coupling (higher $\mathcal{R} a$ ) for $N_{v z}=10$; dotted line: mechanical coupling for $N_{v z}=2$; dashed line: mechanical coupling (preferred) for $N_{v i}=1$. Note that in the $N_{v z}=1$ case, mechanical coupling is preferred, and the lower layer is dragged by the upper one. For $N_{v_{z}}=10$, thermal coupling is preferred; it "works" with low velocity in the lower layer, while mechanical coupling for the same $N_{v z}$ needs a higher Rayleigh number, which allows for higher velocity in the lower layer.

was also observed for this pair, although interface tension is very small, and all properties are very similar. Overstability is obtained for $d=1$. The effect of interface viscosity is shown in Figures 14 and 15 . In the first figure, we have plotted the marginal stability curves that correspond to 


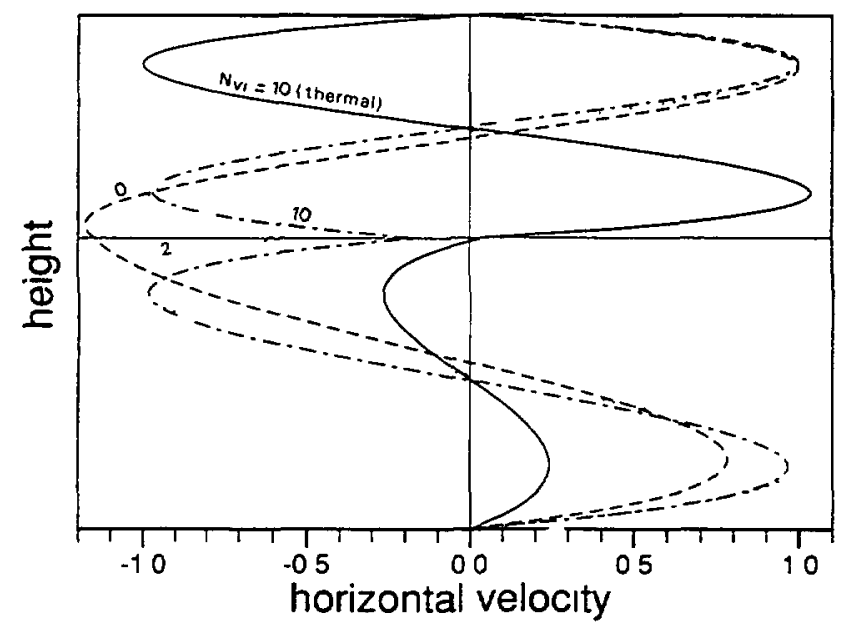

Fig. 13. - Same as in Figure 12, but for horizontal velocity. The different line styles have the same meaning as in Figure 12, except that the dashed line is for $N_{v z}=0$ (preferred mechanical coupling). As $N_{v \imath}$ increases, the horizontal velocity at the interface decreases, which is why thermal coupling becomes more advantageous.

mechanical coupling, and in the second, those that give thermal coupling. The overstable branches are reported in both figures. The results are even simpler than in the silicone oil over glycerol system. We see that the thermal coupling curves move very little, while the mechanical coupling curves move up as the interface viscosity increases. While mechanical coupling is the most critical coupling for $N_{v z}=0$, thermal coupling takes over for $N_{v z}>17$. An interesting phenomenon is observed for $N_{v z}=1:$ the two curves would be superposed, so that their interaction yields overstability (oscillations) over most of the wave number range.

Let us summarize the results with interface viscosity. Without interface viscosity, mechanical coupling is usually preferred over thermal coupling, because it minimizes shear stress at the interface. As the interface viscosity goes up, the interface resembles a thin undeformable film; therefore horizontal velocity vanishes at the interface and the shear stress increases. This has little effect on the thermal coupling case, but it makes mechanical coupling much less advantageous. In fact, thermal coupling is then preferred, because it has better thermal efficiency. This reversal takes place for $N_{v}$, values between 1 and 2 .

\section{Discussion.}

In the first part of this paper, we presented results obtained from mechanical experiments in a layered system. These observations demonstrate that the interface plays a specific dynamical role. We could explain most of the observations by introducing an interface viscosity. Defining the interface viscosity number as

$$
N_{v \imath}=\frac{\nu_{3 n t}}{\nu d}
$$

where $\nu_{2 n t}$ is the interface viscosity, $\nu$ the volumic shear viscosity, and $d$ the thickness of the layer, we estimate that this non-dimensional number is of the order of or larger than 1, for the pairs of liquids we investigated.

In the second part of the paper, we reported on marginal stability computations, which we 


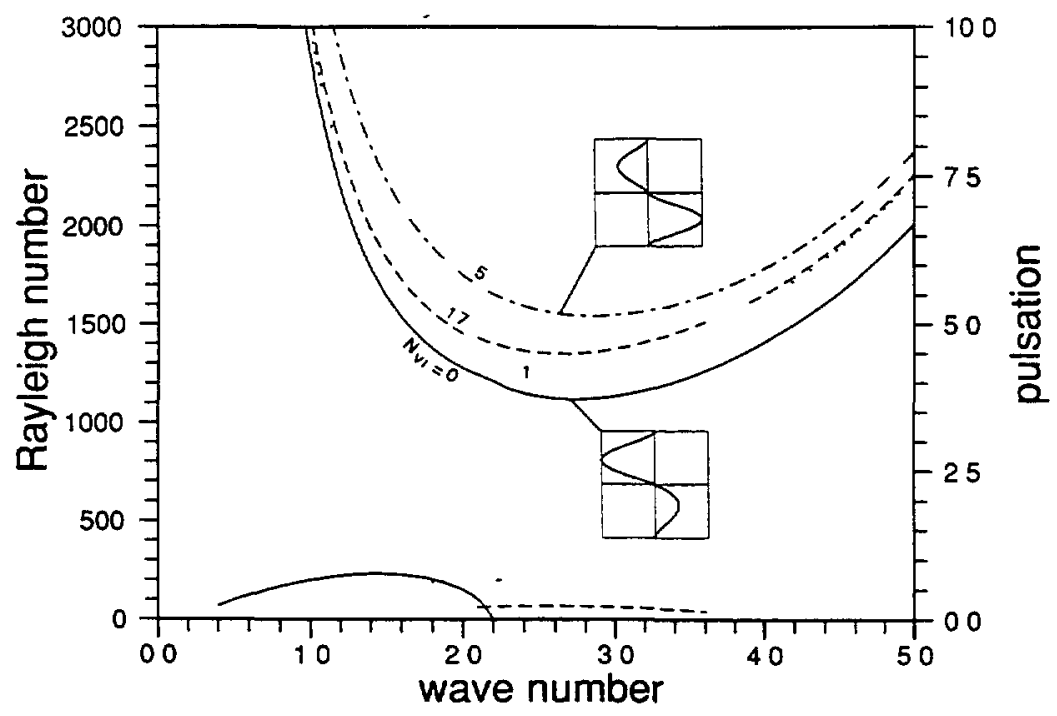

Fig. 14. - Marginal stability in the silicone "light" over silicone "dense" system, with interface viscosity (other paramaters as in Table II). Only the mechanical coupling branches and the overstable ones are shown. The thermal coupling branches are shown in Figure 15. Solid line: $N_{v z}=0$; dotted $N_{v z}=1$; dashed: $N_{v z}=1.7$; chain: $N_{v i}=5$. Note that the overstable region occupies the whole wave number range shown for $N_{v i}=1$. Mechanical coupling requires higher and higher Rayleigh numbers as the $N_{v z}$ number goes up.

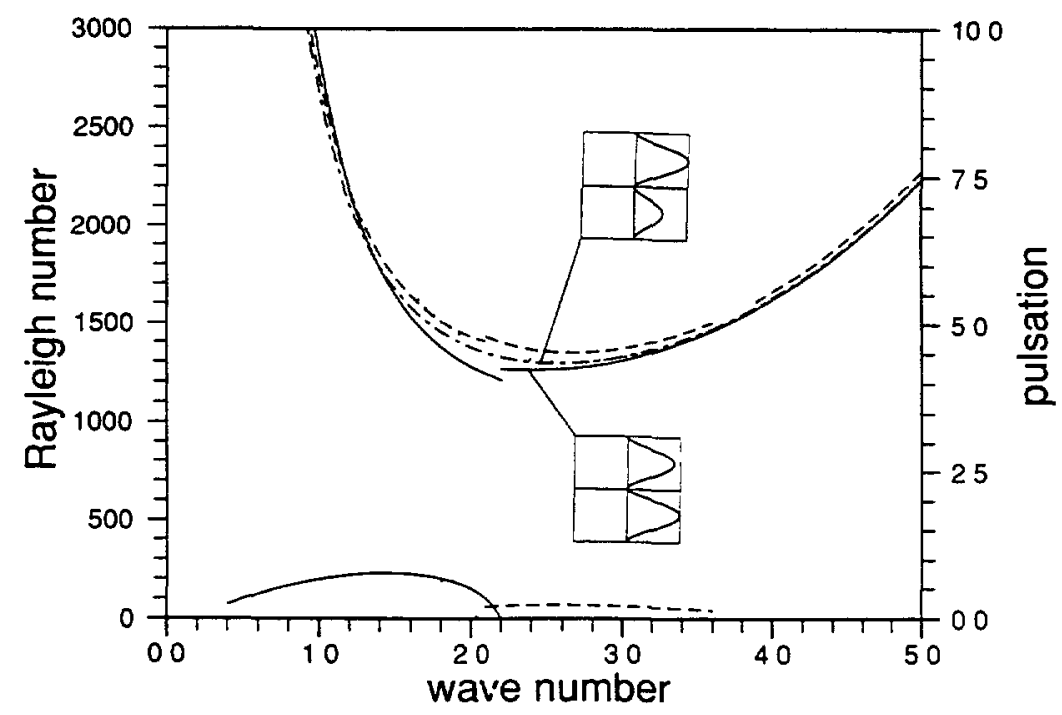

Fig. 15. - Same as Figure 14, but the thermal coupling branches (and the overstable ones) are drawn. Note that the Rayleigh numbers increase only slightly as the $N_{v z}$ number goes up. Thermal coupling is the preferred mode for $N_{v z}>1$.

performed in order to explain the convection experiments described by Nataf et al. [13] and Cardin \& Nataf [14]. These, which were carried out with the pairs of liquids used in the first part of 
the present paper, all show thermal coupling to be the preferred mode of convection. Using the physical properties of these liquids, we computed the marginal stability curves. We found that mechanical coupling should be obtained, even when the effects of interface deformations and temperature-dependent interface tension are included. However, when interface viscosity is introduced, we find that thermal coupling overtakes mechanical coupling when the interface viscosity number $N_{v z}$ exceeds about 1.5 .

Therefore, we think that interface viscosity is present, and is responsible for the thermal coupling observed in the experiments of Nataf et al. [13] and Cardin \& Nataf [14]. Nevertheless, we must keep in mind that the mechanical experiments also give evidence for a concentrationdependent effect. This was not taken into account in the marginal stability analysis. One could have an interface tension that varies with concentration, and hence with the velocity field. Introducing the supplementary concentration equation [8], and assuming that diffusion of the unknown surfactant is small, this would yield a term exactly similar to the interface viscosity term in equation (II.12), except that it contains the time factor $\frac{1}{\sigma P r}$. Its effect, like that of density ratio, is therefore limited to the overstability regime, because the fluctuation of concentration, as well as the interface deformation, is zero in the reference state. It would not yield stationary thermal coupling by itself, whereas interface viscosity does.

However, non linear effects can be important even very close to the threshold of convection [14], so that the role of concentration-dependent interface tension cannot be ruled out.

We must also mention that we do not know which surfactant is responsible for the observed interface viscosity or concentration-dependent interface tension. If its presence, and its concentration, could be better controlled, it would be possible to reduce the $N_{v z}$ number, and to observe mechanical coupling experimentally, or possibly the overstability regime. Alternatively, these regimes could be reached by increasing the volumic shear viscosities and/or the depths of the layers.

\section{Acknowledgements.}

We wish to thank Gilbert Malfait and Guy Marolleau for their skilled technical assistance. The marginal stability analysis was initiated under the guidance of Christiane Normand, and with the help of B. Barriere and J. Couturier. We acknowledge useful discussions with I. Mutabazi and J. Weisfreid, V. Croquette, J. Platten, D. Langevin and P. Vasseur. We are greatly indebted to two anonymous reviewers for very constructive comments, and a thorough proof-reading. This work was suported by CNRS and INSU. Contribution INSU-DBT $n^{\circ} 284$, and ENS-PARIS-Geologie $n^{\circ} 139$.

\section{References}

[1] RICHTER F.M. and MCKENZIE J. Geophys. Res. 86 (1981) 6133-6142

[2] PROCTOR M.R.E. and JONES C.A., J. Flud Mech. 188 (1988) 301-335.

[3] RICHTER F.M. and JOHNSON C.E., J. Geophys Res 79 (1974) 1635-1639.

[4] HONDA S., Bull. Earthquake Res. Inst. 57 (1982) 273-302

[5] BUSSE F.H., Phys. Earth Planet. Int. 24 (1981) 320-324.

[6] BuSSE F.H., Geophys. Res. Lett. 9 (1982) 519-522.

[7] ZEREN R.W. and REYNOLDS W.C., J. Fluid Mech. 53 (1972) 305-327.

[8] WAHAL S. and BOSE A., Phys. Fluids 31 (1988) 3502-3510. 
[9] RASENAT S., BUSSE F.H. and REHBERG I., J. Fluid Mech 199 (1989) 519-540.

[10] CSEREPES L. and RABINOWICZ M., Earth Planet. Sci Lett. 76 (1985) 193-207.

[11] ELLSWORTH K. and SCHUBERT G., Geophys. J. 93 (1988) 347-363.

[12] CSEREPES L., RABINOWICZ M. and ROSEMBERG-BOROT C., J. Geophys. Res. 43 (1988) 12005-12025.

[13] NATAF H.C., MORENO S. and CARDIN Ph., J. Phys. France 49 (1988) 1707-1714.

[14] CARDIN Ph. and NATAF H.C., Europhysics Lett. 14 (1991) 655-660.

[15] LaNGevin D., J. Coll. Int. Sci 80 (1981) 412-425.

[16 HUhNERfuSS H., J. Coll Int. Sci 107 (1985) 84-95.

[17] HASSAGER O. and WestBorg H., J. Coll. Int. Sci. 119 (1987) 524-530.

[18] RUTHERFORD A., Vectors, tensors and the basic equations of fluid mechanics, Prentice Hall Int. (1962).

[19] VILA M.A., KUZ V.A. and RODRIGUEZ A.E., J. Coll Int. Sci. 107 (1985) 314-321.

[20] CLIFT R., GRACE J.R. and WEBER M.E., Bubbles, drops and particles, Academic Press (1978).

[21] LANDAU and LIFCHITZ, Mécanique des fluides, Edıtıons MIR (1971).

[22] SAVIC P., Nath Res. Counc. Can. MT 22 (1953)

[23] CHANDRASEKAR S., Hydrodynamic and Hydromagnetıc stability, Clarendon Oxford (1961).

[24] KRUEGER R., CROSS A. and DIPRIMA R.C., J. Fhuld Mech. 24 (1966) 521.

[25] Mutabazi I., Normand C., Peerhossaini H. and Weisfreid J.E., Phys. Rev. $A 39$ (1989) 763-771.

[26] Pellew A. and SOUTHWELL R.V., Proc. R. Soc. Lond A 176 (1940) 312-343.

[27] VILlERS D. and PLATTEN J.K.,Appl Sci. Res. 45 (1988) 145-152. 